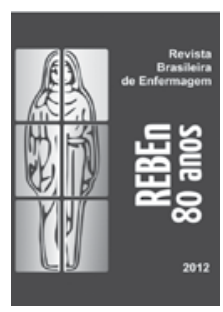

\title{
Lições aprendidas com o trabalho em Rede em Enfermagem e Obstetrícia
}

\author{
Lessons learned with the work as a Network in Nursing and Midwifery \\ Lecciones aprendidas con el trabajo en Red en Enfermería y Partería
}

\begin{abstract}
Isabel Amélia Costa Mendes', Carla Aparecida Arena Ventura", Maria Auxiliadora Trevizan', Lívia de Oliveira Pasqualin"', Sílvia Helena Tognoli'", Juliana Gazzotti'

' Universidade de São Paulo, Escola de Enfermagem de Ribeirão Preto, Departamento de Enfermagem Geral e Especializada. Ribeirão Preto-SP, Brasil.

"Universidade de São Paulo, Escola de Enfermagem de Ribeirão Preto, Departamento de Enfermagem Psiquiátrica e Ciências Humanas. Ribeirão Preto-SP, Brasil.

I'I Fundação Oswaldo Cruz, Escola Nacional de Saúde Pública Sérgio Arouca,

Mestrado Profissional em Saúde Global e Diplomacia da Saúde (Mestranda). Rio de Janeiro-RJ, Brasil.

Iv Universidade de São Paulo, Escola de Enfermagem de Ribeirão Preto, Departamento de Enfermagem Geral e Especializada,

Programa de Pós-Graduação em Enfermagem Fundamental (Mestranda). Ribeirão Preto-SP, Brasil.
\end{abstract}

Submissão: 04-06-2013 Aprovação: 06-06-2013

\section{RESUMO}

A Rede Global de Centros Colaboradores da OMS para o Desenvolvimento da Enfermagem e Obstetrícia é uma organização independente, internacional, sem fins lucrativos, composta por 44 Centros Colaboradores. Dentre seus membros estão líderes de enfermagem e obstetrícia reconhecidos internacionalmente, o que ressalta o significado ímpar deste grupo. Com base em sua trajetória, este artigo descreve como o trabalho em rede pode transformar ações isoladas com resultados pontuais em ações convergentes e sinérgicas com resultados expandidos, e impacto na academia, serviços e arena política.

Descritores: Enfermagem; Obstetrícia; Rede; Liderança em Enfermagem.

\section{ABSTRACT}

The Global Network of WHO Collaborating Centres for Nursing and Midwifery Development is an independent, international, non-profit organization, with 44 Collaborating Centres. Among its members there are internationally recognized nursing and midwifery leaders, what emphasizes the unique meaning of this group. Based on its history, this article describes how the work as a network can transform isolated actions with specific results in convergent and synergic actions with expanded results and impact in academia, services and the political arena.

Key words: Nursing; Midwifery; Network; Nursing Leadership.

\section{RESUMEN}

La Red Global de Centros Colaboradores de la OMS para el Desarrollo de la Enfermería y Obstetricia es una organización independiente, internacional, sin fines lucrativos, formada por 44 Centros Colaboradores. Entre sus miembros están los líderes de enfermería y partería reconocidos internacionalmente, lo que resalta el significado impar de este grupo. Con base en su historia, este artículo describe como el trabajo en red puede transformar acciones aisladas con resultados puntuales en acciones convergentes y sinérgicas con resultados expandidos e impacto en la academia, servicios y arena política.

Palabras clave: Enfermería; Partería; Red; Liderazgo en Enfermería. 


\section{INTRODUÇÃO}

As diretrizes políticas com foco no bem estar social, estruturadas no século XX, estão diretamente relacionadas a mecanismos de incentivo à colaboração entre diferentes organizações com a promoção de trabalho conjunto que vise lidar com problemas complexos e de difícil resolução por uma organização isolada ${ }^{(1)}$. Considerando esta realidade, a Organização Mundial da Saúde (OMS) passou a promover a política de designação de Centros Colaboradores, com planos de ação específicos e delimitados a sua localidade, com o propósito de contribuir com o mandato da OMS em sua área de atuação.

Nesse cenário, com o passar do tempo, consolidou-se uma identidade de grupo entre os Centros Colaboradores que, incentivados pela própria OMS, perceberam a possibilidade de expansão de seu espectro de atividades por meio da valorização da colaboração entre eles. Foi criada, então, em 1988, a Rede Global de Centros Colaboradores da Organização Mundial da Saúde (OMS) para o Desenvolvimento da Enfermagem e Obstetrícia.

A Rede Global é uma organização independente, internacional, sem fins lucrativos, composta, atualmente, por 44 Centros Colaboradores. Dentre seus membros estão líderes de enfermagem e obstetrícia reconhecidos internacionalmente, o que ressalta o significado ímpar deste grupo.

A missão da Rede Global é maximizar a contribuição da Enfermagem e Obstetrícia para o avanço dos Estados, Centros membros, Organizações Não Governamentais e outros interessados na promoção da saúde das populações. A Rede desenvolve atividades de advocacia e políticas baseadas em evidências no âmbito das diretrizes da Assembleia Mundial da Saúde, resoluções regionais e o Programa de Trabalho da OMS. Sua visão é "saúde para todos por meio da excelência da Enfermagem e Obstetrícia"(2).

A Rede Global é administrada por uma Secretaria Geral e Comitê Executivo, composto por um Centro Colaborador representante de cada uma das seis regiões de trabalho da OMS. A Secretária Geral atua como Presidente do Comitê Executivo. De 1988 a 2008, a Secretaria esteve sediada nos seguintes Centros Colaboradores: Universidade de Illinois em Chicago nos EUA, Universidade Yonsei na Coréia do Sul, Universidade de Manchester na Inglaterra, Universidade George Mason nos EUA e Universidade Glasgow Caledonian na Escócia. De 2008 a 2014 está sediada no Centro Colaborador da OMS para o Desenvolvimento da Pesquisa em Enfermagem da Escola de Enfermagem de Ribeirão Preto da Universidade de São Paulo, Centro este que representa o Brasil na referida Rede desde sua fundação ${ }^{(3-5)}$

A missão assumida por esta Secretaria é fortalecer e incrementar a estrutura e o trabalho desenvolvido pelas Secretarias anteriores e unir os Centros Colaboradores em atividades comuns focando os objetivos da OMS e favorecendo sua sinergia ${ }^{(2)}$.

As áreas de trabalho desta Secretaria são: visibilidade - demonstrar a importância do que a Enfermagem e Obstetrícia fazem para melhorar as condições de saúde das pessoas no mundo; ação - trabalho conjunto em projetos para fortalecer as atividades de enfermagem, especialmente na área dos recursos humanos; parcerias - construir relacionamentos com diferentes stakeholders visando o desenvolvimento de projetos globais ${ }^{(2)}$.

Este artigo descreve como o trabalho em rede pode transformar ações isoladas com resultados pontuais em ações convergentes e sinérgicas com resultados expandidos e impacto na academia, serviços e arena política.

\section{REDE GLOBAL DE CENTROS COLABORADORES DA OMS PARA A ENFERMAGEM E OBSTETRÍCIA}

O trabalho em rede ocorre por meio de processos sociais tendo em vista a consecução de fins comuns, e a sua coordenação apresenta como maior desafio facilitar o relacionamento em rede com o propósito de manter a produção conjunta de seus membros. Sendo assim, o foco é a colaboração tanto no que se refere a ideias, como nas interações ${ }^{(6)}$.

Nessa perspectiva, apesar de um incentivo cada vez maior à criação e implementação de redes, verificam-se lacunas relacionadas a evidências empíricas sobre sua real efetividade e atuação, o que, muitas vezes, limita a contribuição da academia para o desenvolvimento de teorias sobre o tema ${ }^{(7-8)}$. Sendo assim, o presente artigo apresenta duas motivações: além de divulgar o trabalho em rede realizado na enfermagem, contribuir, também, com base na experiência prática das autoras, com elementos que agreguem ao debate sobre benefícios e desafios vivenciados com o trabalho em rede, especialmente em um contexto multicultural como é o da Rede Global.

A Rede Global é formada atualmente por 44 Centros Colaboradores, distribuídos nas seis regiões de trabalho da OMS: 19 na região das Américas (AMRO), 3 na região africana (AFRO); 5 na região da Europa (EURO), 2 na região leste do Mediterrâneo (EMRO), 6 na região do Sudeste Asiático (SEARO) e 9 na região ocidental do Pacífico (WPRO), como meIhor se visualiza no mapa abaixo.

A Rede Global apresenta, portanto, uma grande diversidade de abrangência geográfica e de culturas. Nesse sentido, um dos grandes desafios enfrentados é a aderência da estrutura institucional interna dos membros com a estrutura da rede ${ }^{(9-10)}$.

Como uma das estratégias criadas para lidar com esta grande variedade interna e considerando a missão desta Secretaria da Rede Global, foi desenvolvido um plano de ação com os seguintes objetivos: fortalecer e intensificar a comunicação entre os membros; rever a estrutura organizacional da Rede e revisar o seu Planejamento Estratégico. Visando concretizar estes objetivos, a Rede implementa, monitora e avalia continuamente uma série de atividades, de acordo com um cronograma de trabalho pré-estabelecido e aprovado por seus membros ${ }^{(2)}$.

Dessa forma, esta Secretaria busca fortalecer o relacionamento entre os membros da rede, enfatizando a interdependência entre suas ações internas e as oportunidades de colaboração com a OMS e os outros Centros Colaboradores. Nesse contexto, a ideia dos Centros Colaboradores de trabalharem em conjunto por meio da implementação de uma rede representou uma alternativa viável para fortalecer nossa liderança em diferentes atividades e esferas de decisão. Em décadas recentes, as redes continuamente aparecem como 
Figura 1 - Distribuição dos 44 Centros Colaboradores da Organização Mundial da Saúde para o desenvolvimento da Enfermagem e Obstetrícia, segundo ordem cronológica da primeira designação e região da OMS.

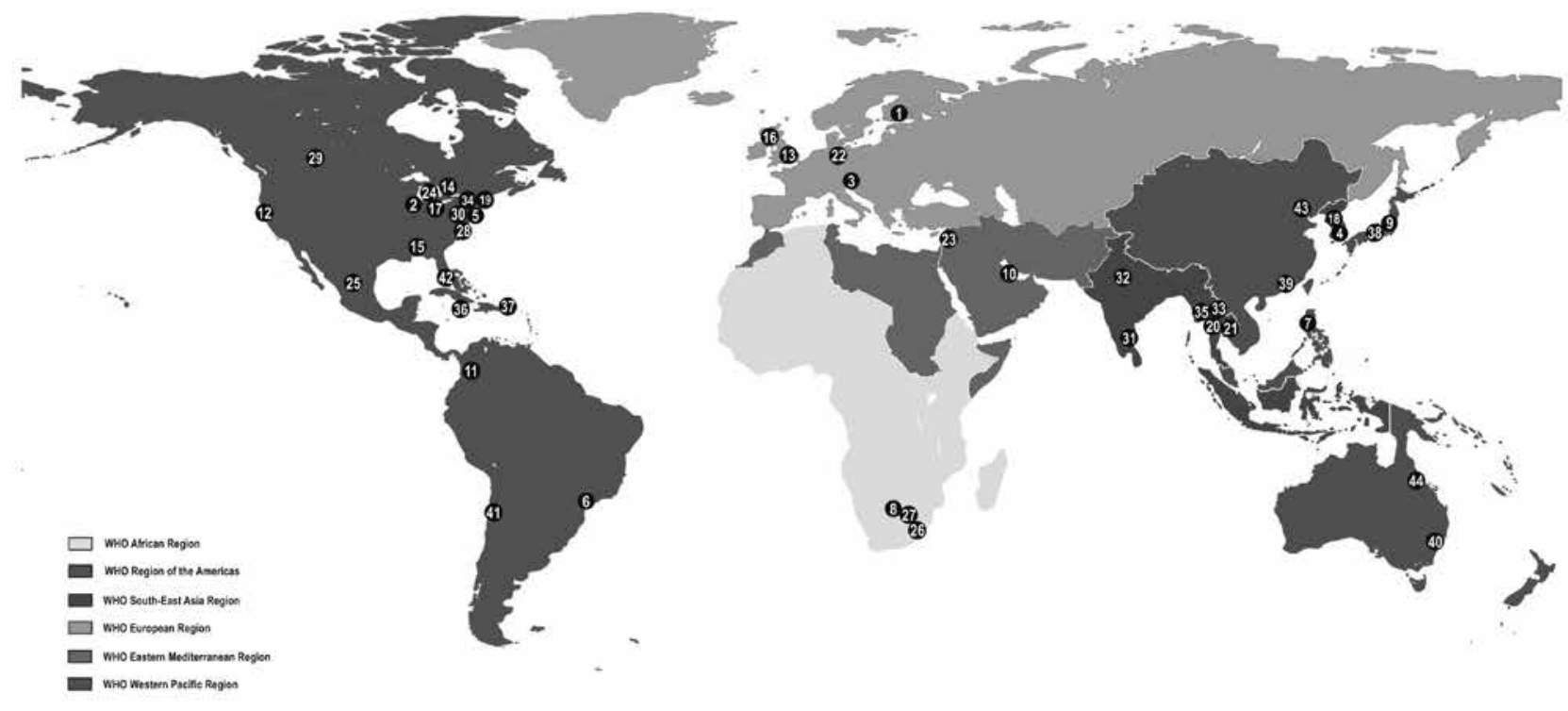

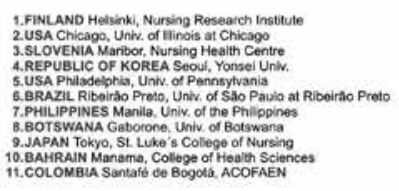

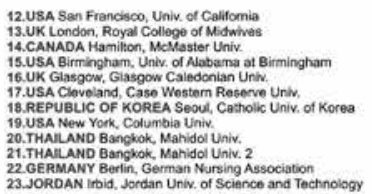

uma das inovações humanas mais significativas no campo das organizações sociais. No mundo científico, tradicionalmente intensivo em redes colaborativas, que operam localmente, regionalmente, nacionalmente e internacionalmente, esta estratégia tem sido utilizada como meio de troca e compartilhamento de informações, articulação política, implementação de ações conjuntas, estabelecimento de estratégias de cooperação e colaboração em pesquisa, dentre outros objetivos.

A proposta de trabalho em rede se apresenta como uma alternativa viável de fortalecimento da liderança da enfermagem e obstetrícia em diferentes esferas de atuação e decisão. Desse modo, o plano de ação desta Secretaria busca o equilíbrio entre as ligações de coesão e dispersão entre seus membros, valorizando a unificação e o fortalecimento de laços, bem como a descentralização no processo de tomada de decisão ${ }^{(11)}$, por meio do Comitê Executivo da Rede.

As estratégias delineadas e desenvolvidas pela Secretaria da Rede Global no período de 2008 a 2012, suas potencialidades e barreiras são descritas a seguir.

\section{Formação e Desenvolvimento de Recursos Humanos em Enfermagem e Obstetrícia}

A Secretaria da Rede Global realizou um mapeamento das áreas comuns de trabalho dos Centros Colaboradores com o propósito de conhecer o que efetivamente é desenvolvido pelos Centros e aproximar Centros que atuam em áreas comuns $^{(12)}$. Considerando ser a estrutura e eficiência da Rede
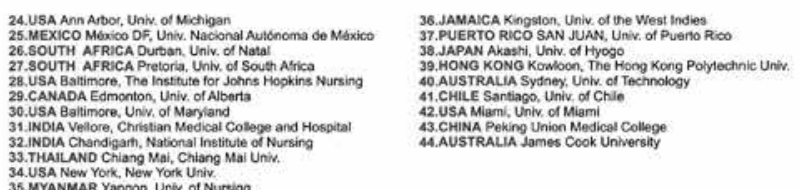

34. USA New York, New M Yok U Univ
35. MYANMAR Yangon, Unk of Nuring

predominantemente endógena, os resultados de seu movimento de evolução serão mais efetivos à medida que sua atuação consiga valorizar a diversidade existente entre seus membros, bem como sua conexão contínua e sistemática ${ }^{(13)}$.

Desse modo, o mapeamento levou à clara percepção de que a formação de recursos humanos em Enfermagem e Obstetrícia representa ponto focal do trabalho desenvolvido pelos Centros, uma vez que a maioria destas organizações $(88 \%)$ são Escolas ou Faculdades de Enfermagem.

Os Centros Colaboradores são referência no ensino de graduação e pós-graduação (lato sensu e stricto sensu) em enfermagem e obstetrícia, e como tal, desempenham um papel catalizador de futuras lideranças internas e estrangeiras, que buscam nestes Centros formação sólida e exemplos de liderança para que possam atuar como agentes de mudanças de suas realidades nacionais e regionais.

Dessa forma, por congregarem lideranças com diferentes formações e experiências, os membros da Rede atuam como pólo dinamizador de influência e intercâmbio entre enfermeiros e obstetrizes das diferentes regiões da OMS.

Outra estratégia de aumento da visibilidade das ações dos Centros Colaboradores é a publicação bianual da Revista Nursing and Midwifery Links. Esta revista foi criada pelas Secretarias anteriores com o objetivo de disseminar informações sobre a rede e publicar artigos técnico-científicos relacionados à Enfermagem e Obstetrícia. Até o momento, esta Secretaria publicou seis volumes da revista, divulgando as 
ações dos membros da Rede e de seus parceiros.

Além da revista, esta Secretaria idealizou e implementou a publicação quinzenal da Newsletter da Rede Global, com informações atualizadas sobre atividades desenvolvidas na sede da OMS, em suas representações regionais, bem como sobre temas atuais de interesse para a saúde, enfermagem e obstetrícia.

A Rede organiza e realiza, ainda a cada dois anos, logo após a Reunião presencial do Comitê Executivo e a Reunião Geral de seus membros, uma Conferência Científica. A estratégia de criação deste espaço representa um meio de interação da rede com a sociedade, beneficiando os profissionais e ampliando a visibilidade do conhecimento gerado pela enfermagem e obstetrícia e a sua integração com áreas da saúde e afins. Em 2010, esta Secretaria organizou, em conjunto com o Centro Colaborador para o Desenvolvimento da Pesquisa em Enfermagem do Brasil, a $8^{\text {a }}$ Conferência, intitulada "Atenção Primária à Saúde: muitas perspectivas, uma meta", que contou com 1195 membros efetivos de 35 países e das seis regiões de trabalho da OMS. Em 2012, foi realizada a segunda edição do evento durante a gestão desta Secretaria em Kobe, Japão, tendo como tema central "Mesmo com cuidados básicos, prepare-se para o inesperado", organizada em conjunto com o Centro Colaborador da Universidade de Hyogo.

Além destas ações coordenadas, os membros da Rede Global são chamados a participarem de grupos de trabalho e Comissões criadas pela OMS para o desenvolvimento de materiais e publicações especialmente voltados para a formação de recursos humanos em suas distintas áreas de especialidade.

Observa-se, portanto, que os membros da Rede possuem um papel importante na formação direta de recursos humanos, assim como na instrumentalização desta formação, por meio da produção e da disseminação das lições apreendidas como uma organização que promove a busca de excelência no ensino, e valoriza a internacionalização e a integração de seus membros, instituições e regiões.

O papel político dos Centros Colaboradores e da Rede Global no fortalecimento das lideranças de Enfermagem e Obstetrícia

Quanto à contribuição dos Centros Colaboradores membros da Rede Global em diferentes instâncias de decisão em saúde e suas interfaces, nas esferas municipal, estadual e federal/nacional, o fato de serem reconhecidos como referência por uma Organização Internacional, como a OMS, fortalece suas possibilidades de participação.

Contudo, a participação dos Centros Colaboradores, na maioria dos casos, ocorre predominantemente na implementação de programas e ações, ao invés de se dar também nos processos de articulação política e tomada de decisão. Apesar de avanços, observam-se, ainda, lacunas na valorização externa e do próprio profissional de enfermagem e obstetrícia, de seu conhecimento, expertise e potencial no desenho de políticas e programas para fortalecimento dos sistemas de saúde.

A Rede realiza reuniões anuais e, através delas, alcança os objetivos de compartilhar experiências bem sucedidas de atuação de enfermeiros e obstetrizes nas decisões em saúde, demonstra o papel efetivo que o enfermeiro e obstetriz podem desempenhar e cultiva a consciência interna sobre estas possibilidades ${ }^{(14)}$ Estimula-se, nesse contexto, a participação destes profissionais em diferentes órgãos governamentais e não governamentais.

É importante destacar que a consolidação deste sentido de força da enfermagem e obstetrícia por seus próprios profissionais se intensifica em suas relações com os pares ${ }^{(15)}$, por meio da participação em órgãos reguladores e sociedades científicas das profissões.

Além de ações voltadas para o cenário interno, esta Secretaria busca fortalecer sua parceria com organizações-chave para a enfermagem e obstetrícia, como o International Council of Nurses, International Council of Midwives, a Sigma Theta Tau International e a Global Alliance for Nursing and Midwifery. Não se pode deixar de mencionar a participação da Rede em fóruns de discussão e tomadas de decisões globais para a enfermagem e obstetrícia organizados pela OMS, como, por exemplo, o Global Advisory Group for Nursing and Midwifery. A possibilidade de participar como observador demonstra o reconhecimento destas profissões e potencial de atuação desta Rede. Por outro lado, ilustra aos membros caminhos que podem ser buscados para esses profissionais exercitarem o papel político que podem desempenhar, reivindicando sua inclusão em processos decisórios, além de facilitar a seus membros o acesso a documentação privilegiada sobre discussões atualizadas em saúde.

Nesse sentido, a contribuição da rede poderia ser mais efetiva se contasse, em sua composição, com Centros Colaboradores representantes de instituições de serviços de saúde, nas áreas de enfermagem e obstetrícia. Outro ponto a ser trabaIhado é o incentivo a designação pela OMS de mais Centros Colaboradores nas diferentes regiões da OMS.

\section{Desafios para a efetivação do trabalho em rede entre os Centros Colaboradores e ações desenvolvidas}

Considerando a heterogeneidade dos Centros Colaboradores, sua diversidade cultural e de experiências regionais, existem muitas barreiras a serem transpostas conjuntamente para a consolidação do trabalho em rede. Os membros da Rede têm ciência destas dificuldades e vêm buscando alternativas para enfrentar estes desafios.

Dessa forma, com base na avaliação positiva da experiência da Rede Global, os membros decidiram formar redes regionais. Outra alternativa adotada pela atual Secretaria da Rede para realizar ações consideradas chave em seu plano de ação, foi o trabalho em Forças Tarefa. Esta ferramenta busca permitir que os membros dediquem seu tempo, atenção e experiência para atingir objetivos específicos. As forças tarefa incluem sete membros, seis representando cada região da OMS e um Coordenador, membro do Comitê Executivo. As Forças Tarefa devem, com base em sua experiência e conhecimento, entender profundamente os componentes e diagnosticar problemas relacionadas à situações inseridas no plano de ação da Rede. Como resultado, devem propor um plano final de atividades a ser desenvolvido por todos os membros da Rede ${ }^{(2)}$.

Em suma, o histórico de atuação da Rede Global demonstra importantes desafios que estão sendo enfrentados 
gradativamente nestes 25 anos de colaboração. As alternativas buscadas por esta Secretaria desde 2008 e relatadas neste artigo demonstram a sua legitimidade como referencia de liderança entre seus membros e entre a Rede e o seu ambiente externo.

\section{CONSIDERAÇÕES FINAIS}

A criação da Rede Global de Centros Colaboradores da OMS representou relevante marco histórico para a Enfermagem e Obstetrícia, fortalecendo ainda mais a visibilidade dessas profissões junto à OMS e de seus membros nos cenários nacional e internacional.

Este artigo descreveu a experiência da atual Secretaria da Rede Global que, desde 2008, desenvolve estratégias visando potencializar os resultados das ações de seus membros por meio de um efetivo trabalho em rede.
Apesar de ausência de estudos empíricos sobre o trabalho em rede, as ações desenvolvidas e seus resultados de curto-prazo apontam a relevância da existência de objetivos comuns e, especialmente, do estabelecimento de relações de confiança entre seus membros, que se refletem na legitimidade de sua Secretaria e Comitê Executivo. Além disso, a valorização de experiências prévias de atuação isolada e de ações conjuntas de seus membros também promovem a comunicação aberta e o comprometimento de cada elo desta engrenagem com o compartilhamento de informações.

As vivências relatadas, assim como as estratégias adotadas e exploradas neste trabalho podem iluminar os caminhos de outras redes internacionais em formação. Nesse contexto, é fundamental que a liderança da rede explore ao máximo sua diversidade de maneira construtiva buscando, simultaneamente, consolidar uma identidade de rede com foco na interação e colaboração entre seus membros.

\section{REFERÊNCIAS}

1. Dunlop JM, Holosko MJ. The story behind the story of collaborative networks - relationships do matter! J Health Soc Policy 2004;19(3):1-18.

2. Mendes IAC, Ventura CAAV. Plan of action global network of WHO collaborating centres for nursing and midwifery. Nurs Midwifery Links 2009;1(1):5-10.

3. Mendes IAC, Ventura CAA, Trevizan MA. O Centro colaborador da OMS para o desenvolvimento da pesquisa em enfermagem e suas parcerias internacionais. Hist Enferm Rev Eletronica 2010;1:150-162.

4. Mendes IAC, Trevizan MA. Ações do centro colaborador da OMS para o desenvolvimento da pesquisa em enfermagem: o caso brasileiro. Acta Paul Enferm 2000;13(2):9-15.

5. Mendes IAC, Silveira IMB, Ventura CAA. 1998: EERP/USP - 45 anos, 10 anos como centro colaborador da OMS para o desenvolvimento da pesquisa em enfermagem. Informativo Latino-Americano de Enfermagem 1998;24:1.

6. O'Toole LJ. The implications for democracy in a networked bureaucratic world. J Public Adm Res Theory 1997; 7(3):443-59.

7. Grahan JR, Barter K. Collaboration: a social work practice mothod. Families in society: the Journal of Contemporary Human Services 1999;80(1)6-13.

8. Rubin HJ, Rubin IS. Community organizing and development. New York: MacMillan; 2001.
9. García-Ramírez M, Paloma V, Suarez-Balcazar Y, Balcazar F. Building International Collaborative Capacity: contributions of community psychologists to a European Network. Am J Community Psychol 2009;44(1-2):116-122.

10. Wellman B. Network Analysis: some basic principles. Sociological Theory 1993;1(1):155-200.

11. Pallotti F, Lomi A, Mascia D. From network ties to network structures. Exponential random graph models of interorganizational relations. Qual Quant 2013;47(3):1665-85.

12. Mendes IAC, Ventura CAAV. Global Network of WHO collaborating centres for nursing and midwifery - Report 2008-2010. Ribeirão Preto: Global Network of WHO Collaborating Centres for Nursing and Midwifery Development; 2010.

13. Cárdenas J. Varieties of corporate networks: network analysis and fsQCA. International J Comparative Sociol 2012;53(4):298-322.

14. Mendes IAC, Ventura CAAV. Global Network of WHO Collaborating Centres for Nursing and Midwifery - Report 2010-2012. Ribeirão Preto: Global Network of WHO Collaborating Centres for Nursing and Midwifery Development; 2012.

15. Nowell B. Profiling capacity for coordination and systems change: the relative contribution of stakeholder relationships in interorganizational collaboratives. Am J Community Psychol 2009;44(3-4):196-212. 